\title{
Spectral Doppler Study of Hemodynamic Alterations in Central Retinal Artery (CRA) and Posterior Ciliary Artery (PCA) in Diabetics
}

\author{
Mahesh ${ }^{1}$, Aishwarya K C ${ }^{2}$, Shrunga Tejasvi ${ }^{3}$, Prasanthi ${ }^{4}$, Prajnha U $\mathbf{P}^{5}$ \\ ${ }^{1}$ Senior Resident, Department of Radio Diagnosis, ${ }^{2}$ Associate Professor, Department of Radio Diagnosis, KVG Medical College \\ and Hospital, ${ }^{3}$ Consultant, Department of Radiologist, Prima Diagnostic Centre, Jayanagar, Bengaluru, ${ }^{4}$ Assistant Professor, \\ Department of Radio Diagnosis, SRMC Medical College, Chennai, ${ }^{5} \mathrm{~K}$ S Hegde Institute of Medical Scienes, Deralakatte, \\ Mangalore, India
}

Corresponding author: Mahesh, Senior Resident, Dept of Radio Diagnosis, Father Muller Medical College and Hospital, Kankanady, Managaluru-575002, India

DOI: http://dx.doi.org/10.21276/ijcmsr.2019.4.2.10

How to cite this article: Mahesh, Aishwarya K C, Shrunga Tejasvi, Prasanthi, Prajnha U P. Spectral doppler study of hemodynamic alterations in central retinal artery (CRA) and posterior ciliary artery (PCA) in diabetics. International Journal of Contemporary Medicine Surgery and Radiology. 2019;4(2):B43-B46.

\section{A B S T R A C T}

Introduction: Estimation of orbital blood flow velocity from Doppler of the CRA and PCA could yield promising results when used in the identification of early retinopathy in diabetics. The aim of this study is to measure the blood flow parameters in CRA and PCA of the eye with Doppler in diabetes and to compare with controls.

Material and methods: Doppler was performed 200 individuals (100 diabetics and 100 controls) in a randomized blinded format. The peak systolic velocity (PSV), end diastolic velocity (EDV), resistance index (RI) of the CRA and the PCA were measured in both groups. Dilated fundoscopy was done for the diabetics for correlation. The data was analyzed using ANOVA and student T test (" $p$ " value $<0.05$ )

Results: As compared with the controls, both the CRA and PCA in diabetics have slower velocities and significantly higher RI. In the CRA of diabetics there was a statistically significant decrease in both the PSV and EDV as compared to the controls. The RI of the CRA was increased in diabetics as compared to the controls. Similarly, there was a decrease in both the PSV and EDV of the PCA in diabetics compared to the control group. The RI of PCA was significantly increased in diabetics as compared to the controls.

Conclusion: Significant changes in retrobulbar blood flow are found in eyes with diabetic retinopathy which can be detected by colour Doppler and it could serve as a promising modality to assess hemodynamic changes in different stages of diabetic retinopathy.

Keywords: Central Retinal Artery, Posterior Ciliary Artery, Orbital Doppler, Diabetes, Retinopathy

\section{INTRODUCTION}

Among the various non communicable diseases, diabetes mellitus(DM) has become a frontrunner and is perhaps as old as mankind itself. ${ }^{1}$

Currently DM has attained the status of a modern day epidemic. According to WHO, India has the highest number of people with diabetes in the world which is set to be a staggering 80 million by the year $2030 .^{2}$ Diabetic retinopathy (DR) is the most common ocular complication in patients with diabetes. ${ }^{3}$ The reported prevalence of $\mathrm{DR}$ varies between studies, but it is probably up to $40 \% .^{3}$ It is more common in type 1 than type 2 diabetes. ${ }^{3}$

Despite extensive research, our knowledge of the effects of diabetes on the ocular circulation is still incomplete. ${ }^{4}$ Research has suggested that most patients with diabetes develop characteristic abnormalities of retinal blood vessels. These abnormalities seem to disturb the retinal microcirculation and reduce the retinal blood flow if not compensated by regulatory mechanisms. Based on the above findings, great importance should be attached to analysis of the ocular circulation in diabetics. ${ }^{5-9}$ DR being a microangiopathy will ultimately result in retinal ischemia. Thus the hypothesis we put forward is that diabetic patients with or without retinopathy will have haemodynamic alterations in the orbital circulation which can be detected by orbital Doppler. However, the role of haemodynamics in diabetic retinopathy has not been clearly defined. The data obtained from the studies done on DR present with conflicting reports. Orbital blood flow velocities were increased in some studies (MacKinnon et al), but decreased in others (Karami et al) or remain unchanged. ${ }^{4,10}$ So there is an immediate need for clarification on the changes that occur in the orbital blood flow in diabetic patients.

In this study the hypothesis was that the diabetic patients will have haemodynamic alterations in the orbital circulation. Orbital Doppler imaging presents an excellent method 
for assessment of orbital blood vessels. It has been used in ophthalmology as a safe and noninvasive modality. It allows us to easily assess blood flow velocity information. Therefore estimation of orbital blood flow velocity from doppler imaging of the central retinal artery (CRA) and posterior ciliary artery (PCA) could yield promising results when used in the identification of early retinopathy in diabetic patients. ${ }^{10}$ So we designed a study with the purpose of measuring the blood flow velocities and resistance index in the CRA and PCA with Doppler sonography in patients with diabetes and to compare the results with those of their non-diabetic peers.

\section{MATERIAL AND METHODS}

The study was conducted in KVG Medical College, Sullia, Karnataka ans was started after receiving clearance from the institutional ethics committee. All the subjects were informed about the study in their vernacular language and written consent was taken from them.

It was a prospective comparative study consisting of 100 cases and 100 controls.

The study period was from November 2016 to December 2017. Patients were selected by systematic random sampling. The age group chosen was between 30- 90 years, inclusive of both male and female patients.

The mean age group of males 55.89 was and that of females was 55.03. The mean age group of males was 61.39 in case and 41.62 in control groups. The mean age group of females was 60.39 in case and 49.96 in control groups.

Diabetes was diagnosed by at least 2 fasting plasma glucose measurements above $126 \mathrm{mg} / \mathrm{dl}$.

A brief medical history which included the duration of diabetes, other relevant medical and drug history was obtained. Further laboratory investigations undergone previously like $\mathrm{HbA} 1 \mathrm{c}$ and sugar levels were recorded.

The diabetic subjects were categorized following dilated fundoscopy using the biomicroscopic indirect ophthalmoscope by a single experienced ( 9 years) medical ophthalmologist before the Doppler study. Patients were classified according to the ETDRS report 1991.

Patients who had undergone previous laser photocoagulation and those with a history of any eye disease which may affect blood flow being studied such as ocular inflammation, trauma (3 excluded), congenital abnormality(1 excluded), non-diabetic vascular disease such as retinal vein occlusion or glaucoma (4 excluded) were excluded from the study. Patients with systemic co morbidities that could affect the ocular circulation were also excluded. The inclusion criteria was a known of diabetes mellitus from the diabetic retinopathy clinic. The non-diabetic controls were recruited from hospital staff and voluntary workers.

Colour and spectral Doppler imaging of the PCA and CRA was performed by a single masked observer (3 years experience) using the Voluson 730 Expert ultrasonography and Doppler machine.

Examination technique was explained to the subjects. The patient was placed in a supine position with the eyes closed and gaze directed at the ceiling. To perform Doppler of the orbit, the ultrasound linear transducer (ranging from 5 to $10 \mathrm{MHz}$ ) was placed on the closed eyelids using a coupling gel, ophthalmic methylcellulose. Care was taken during the examination to avoid excess pressure on the globe, which may result in artifact. Scans were made through the eye and orbit in multiple planes to identify the desired structures and vessels. Gray-scale 2D ultrasound image was used to delineate the normal structures initially, including the globe, optic nerve, extraocular muscles, and orbital blood vessels. Intraocular structures including anterior chamber, lens, and vitreous cavity were also visualized. The colour display and waveform distinguished adjacent vessels, such as the central retinal artery and central retinal vein, which lie in close proximity within the optic nerve. The vessels for the study were identified with the help of knowledge of the vascular anatomy.

The first step involved identifying the optic nerve. The CRA can be found most easily within the optic nerve for approximately $12 \mathrm{~mm}$ behind the globe, where it runs a fairly straight course. It usually is located nasal to the adjacent central retinal vein. The transducer was positioned so that CRA flow is red and CRV is blue(Fig 1). The posterior ciliary arteries are found 10 to $20 \mathrm{~mm}$ behind the globe surrounding the optic nerve and are variable in number. (Fig 2)The waveforms are similar to that of the central retinal artery but may be less pulsatile.

Spectral waveforms of desired vessels were obtained by proper sample volume. The parameters including PSV, EDV and RI were calculated automatically by machine software. $\mathrm{RI}$ is given by (PSV - EDV)/PSV.

Results from a single eye were used, chosen randomly by tossing a coin, if both eyes were equally involved.

Statistical Methodology: Following the Doppler study, statistical analysis was made with help of SPSS (Statistical Package for Social Science, Version.10.0.5) package. Mean Doppler parameters were calculated for each artery separately. Data was entered in Microsoft excel and analyzed using SPSS (Statistical Package for Social Science, Version.10.0.5) package. The results were averaged (mean + standard deviation) for continuous data and number and percentage for dichotomous data. The data from the study was analyzed using A one way analysis of variance (ANOVA) and student $\mathrm{T}$ test. In all the above tests " $\mathrm{p}$ " value of less than 0.05 was accepted as indicating statistical significance.

\section{RESULTS}

In this study 100 cases and 100 controls were taken. The mean age of cases was 61.2 years and that of controls was 49.8 years. Since the 'p' value is not significant the cases and controls age groups are comparable.

Our results showed that as compared with the normal control subjects, both the CRA and PCA in diabetic patients have slower velocities and significantly higher resistance indices (Table 1 and 2, figure -2).

\section{DISCUSSION}

The effects of diabetes mellitus on the retrobulbar circulation have been studied with conflicting evidence as to whether blood supplying the eye is increased or decreased. For example, using laser Doppler Patel et al showed an increase in blood flow velocity in type 1 diabetics with untreated 
retinopathy. ${ }^{11}$ In contrast, in studies using CDI, reduced blood flow velocity was found in diabetic patients and it appeared to become further reduced with the progression of retinopathy. ${ }^{11}$ The inconsistency may be explained in part by different techniques and sites of measurement, differences in patient characteristics, definitions of DR, and there may be an element of chance.

Our results showed that as compared with the normal control subjects, both the CRA and PCA in diabetic patients have slower velocities and significantly higher resistance indices (Table 1 and 2).

The CRA is supposed to reflect the vascular changes associated with diabetes more sensitively than the OA. ${ }^{12,13}$ The decrease in blood velocity in the CRA might be due to a

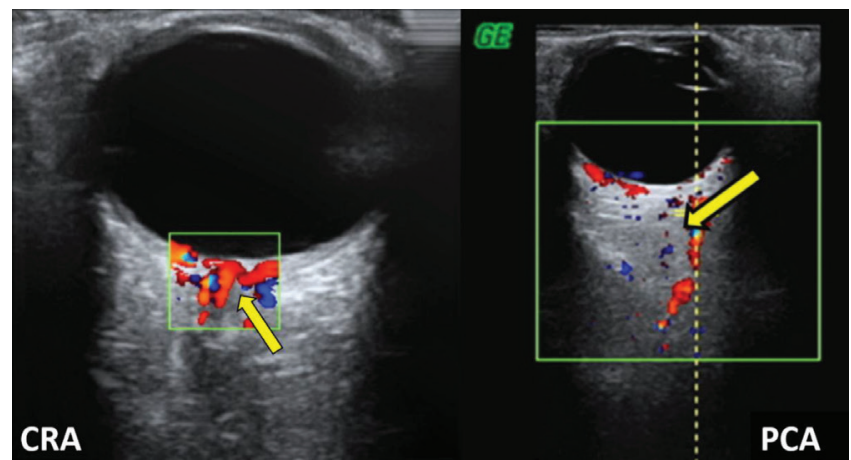

Figure-1: Colour Doppler images showing CRA on right and PCA on left (yellow arrow) changes in the blood and the blood vessels in diabetes such as hypercoagulability, increased stickiness and deformability of the red blood cells, hyperviscosity, thickening of the basement membrane, atherosclerosis and blood vessel tortuosity.

The reduction in blood flow velocity we observed in the CRA is in agreement with laser Doppler velocimetry findings reported by Grunwald et al and metaanalysis by Nana eng et al. Our results also agree with the study from Goebel et al. which found significantly lower PSV and EDV in the CRA of all those with untreated DR compared to controls. ${ }^{14}$ Similar results were found by Mendivil et al. ${ }^{15}$

It has been estimated that of the total ocular blood supply from the OA, more than $90 \%$ is directed to the choroid via the ciliary circulation. The results from this study that show decreased velocities and increased RI in the PCA support the hypothesis that even the choroidal circulation is affected in DR. The PCA's supply the choroid and the optic nerve disc. The histopathological changes of the choroid in diabetes like basement membrane thickening, narrowing of the capillary lumen are correlating with the Doppler findings. One school of thought is that the upregulation of the vascular endothelial growth factor (VEGR) synthesis secondary to choroidal ischemia might trigger the proliferative phase.

The results in the present study regarding the PCA are in agreement with those in previous studies using the pulsatile method that have detected a choroidal circulatory alteration in the DR. The RI is considered to indicate the blood vessel's compliance to changes in blood pressure. In our results the $\mathrm{RI}$ in both CRA and PCA was increased in diabetics.

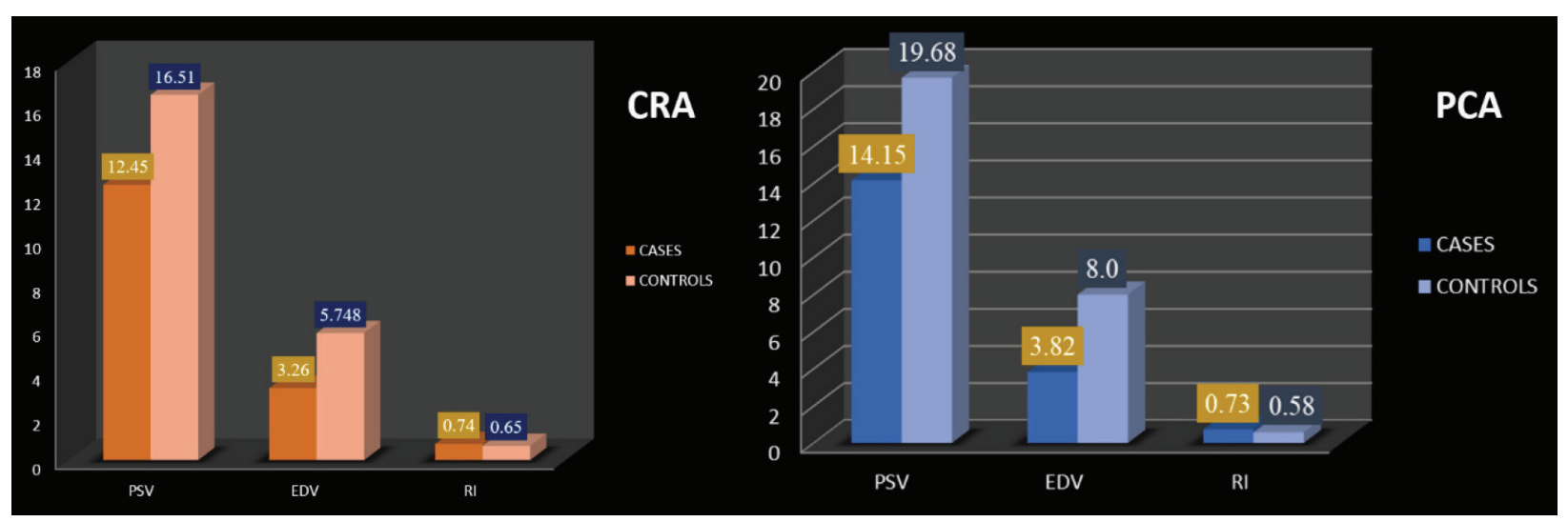

Figure-2: Comparison between the mean Doppler parameters of cases and controls.

\begin{tabular}{|c|c|c|c|c|c|c|c|c|c|}
\hline & \multicolumn{4}{|c|}{ Cases } & \multicolumn{4}{|c|}{ Controls } & \multirow[t]{2}{*}{$P$ value } \\
\hline & Mean & SD & Min & Max & Mean & SD & Min & Max & \\
\hline $\mathrm{PSV}(\mathrm{cm} / \mathrm{sec})$ & 12.45 & 4.38 & 4.72 & 25.06 & 16.51 & 3.2 & 2.1 & 29.6 & $<0.001$ \\
\hline $\mathrm{EDV}(\mathrm{cm} / \mathrm{sec})$ & 3.26 & 1.52 & 9 & 9.1 & 5.74 & 1.68 & 0.69 & 9.88 & $<0.001$ \\
\hline RI & 0.74 & 0.077 & 0.5 & 0.9 & 0.65 & 0.0728 & 0.5 & 1.0 & $<0.001$ \\
\hline
\end{tabular}

\begin{tabular}{|c|c|c|c|c|c|c|c|c|c|}
\hline & \multicolumn{4}{|c|}{ Cases } & \multicolumn{4}{|c|}{ Controls } & \multirow[t]{2}{*}{ P value } \\
\hline & Mean & SD & Min & Max & Mean & SdD & Min & Max & \\
\hline $\mathrm{PSV}(\mathrm{cm} / \mathrm{sec})$ & 14.16 & 5.27 & 2.1 & 29.6 & 19.68 & 5.75 & 9.14 & 39.0 & $<0.001$ \\
\hline $\mathrm{EDV}(\mathrm{cm} / \mathrm{sec})$ & 3.82 & 1.74 & 0.69 & 9.88 & 7.99 & 2.19 & 3.14 & 15.7 & $<0.001$ \\
\hline $\mathrm{RI}$ & 0.73 & 0.082 & 0.5 & 1.0 & 0.58 & 0.0696 & 0.4 & 0.8 & $<0.001$ \\
\hline
\end{tabular}


DM is known to cause changes in the blood like increased blood and plasma viscosities, red blood cell aggregation, platelet aggregation, and platelet shape. Moreover in the eye it causes capillary rarefaction, atherosclerotic changes and leukocyte adhesion. All these pathological changes support this circulatory finding of increased RI. The increased vascular resistivity may further compromise the ocular tissue oxygenation and nourishment and may contribute to the occurrence of neovascularisation.

The above changes in blood flow parameters measured using CDI are probably a consequence of both long-lasting hyperglycemia and glucose level fluctuations in the blood.

Some of the limitation in the present study include that inter observer variability could not be tested and that the study could not establish a statistically significant relationship/ grading between Doppler parameters of the cases with their respective fundoscopic grading (at $\mathrm{p}<0.05$ ). The study being cross sectional, we could not follow up the cases for assessing the changes occurring in the Doppler parameters with the progression of diabetic retinopathy in each individual case.

\section{CONCLUSION}

Altered metabolism in DM results in systemic vascular diseases. Ocular vascular changes and consequently hemodynamic changes play the pivotal roles in the pathogenesis of diabetic retinopathy. However, the effects of diabetes mellitus on the retinal circulation have been studied with conflicting evidence as to whether blood supplying the retina and choroid is increased or decreased. In the current study, we concluded that significant changes in retrobulbar blood flow are found in eyes with diabetic retinopathy and also that colour Doppler imaging is a promising modality to assess hemodynamic changes in different stages of diabetic retinopathy. The findings of reduced velocities in the CRA and the PCA of diabetics with increased RI suggest the involvement of both retinal and choroidalcirculation in the diseased process. Monitoring with colour Doppler imaging may have predictive power in identifying those at greatest risk of developing sight threatening proliferative disease.

\section{REFERENCES}

1. Park K. Parks Textbook Of Preventive And Social Medicine. 22 ${ }^{\text {nd }}$ ed. Jabalpur: M/S Banarasidas Bhanot Publishers; 2013. Chapter 6, Epidemiology of chronic non communicable diseases and conditions; p.334-336.

2. Wild. Global prevalence of diabetes: estimates for the year 2000 and projections for 2030. Diabetes care 2004;27(1):17-53.

3. Kanski JJ, Bowling B. In: Gabbedy R, editor. Clinical Ophthalmology-A Systemic Approach. $7^{\text {th }}$ ed. London: Elsevier publishers; 2011. Chapter 13, Retinal vascular diseases; p.534-550.

4. MacKinnon JR, McKillop G, O’Brien C, Swa K, Butt Z, Nelson P. Colour Doppler imaging of the ocular circulation in diabetic retinopathy. Acta Ophthalmol Scand 2000;78 (5):386-389.

5. Rimmer T, Fleming J, Kohner EM. Hypoxic viscosity and diabetic retinopathy. BRJophthalmol 1990;74 (6):400-04.

6. Tillmann W, Lakomek M, Heidemann P, Behrens-
Baumann W, Schroter W. Aggregate formation of erythrocytes and diabetic retinopathy in children, adolescents, and adults with diabetes mellitus (type I). KlinWochenschr 1984;62 (8):1136-39.

7. Porta M, Hilgard P, Kohner EM. Platelet shape change abnormalities in diabetic retinopathy. Diabetologia 1980;18 (5):217-21.

8. Agardh CD, Agardh E, Bauer B. Platelet aggregation in type I diabetics with and without proliferative retinopathy. Acta Ophthalmol Copenh1987;65 (4):358-62.

9. Soeldner JS, Christacopoulos PS, Gleason RE. Mean retinal circulation time as determined by fluorescein angiography in normal, prediabetic, and chemicaldiabetic subjects. Diabetes 1976;25 (2):903-08.

10. Karami M, Janghorbani M, Dehghani A, Khaksar K, Kaviani A. Orbital Doppler Evaluation of Blood Flow Velocities in Patients with Diabetic Retinopathy. RDS 2012;9 (1):104-111.

11. Ovali GY, Ersoy BB, Tuncyurek OA, Urk V, Ozkol $\mathrm{M}$, Ozhan B et al. Doppler ultrasonography imaging of hemodynamic alteration of retrobulbar circulation in type 1 diabetic children and adolescents without retinopathy. Diabetes research and clinical practice 2008;79 (3):243- 248.

12. Meng N, Liu J, Zhang Y, Jinlan Y, Li H, Qu Y et al. Color Doppler imaging analysis of retrobulbar blood flow velocities in diabetic patients without or with retinopathy a meta-analysis. J ultrasound med 2014;33 (2):1381-1389.

13. Arai T, Numata K, Tanaka K, Kiba T, Kawasaki S, Saito $\mathrm{T}$ et al. Ocular Arterial Flow Hemodynamics in Patients with Diabetes Mellitus. J Ultrasound Med 1998;17 (5):675-681.

14. Goebel W, Lieb W E, Ho A, Sergott R C, Farhoumand R, Grehn F. Color Doppler Imaging: A New Technique to Assess Orbital Blood Flow in Patients With Diabetic Retinopathy. Invest Ophthalmol Vis Sci 1995;36 (1):864-870.

15. Mendivil A, Cuartero V, Mendivil MP. Ocular blood flow velocities in patients with proliferative diabetic retinopathy and healthy volunteers: a prospective study. Br J Ophthalmol 1995. 79 (4):413-416.

Source of Support: Nil; Conflict of Interest: None

Submitted: 10-03-2019; Accepted: 02-05-2019; Published online: 19-05-2019 